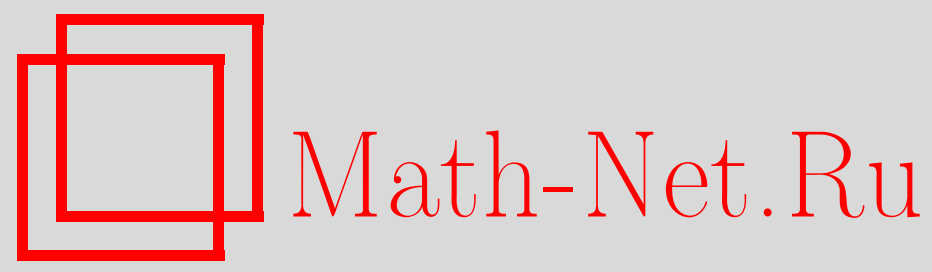

В. П. Заставный, Теорема о нулях целых функций и ее применение, Матем. заметки, 2004, том 75, выпуск 2, 192-207

DOI: https://doi.org/10.4213/mzm23

Использование Общероссийского математического портала Math-Net.Ru подразумевает, что вы прочитали и согласны с пользовательским соглашением http://www. mathnet.ru/rus/agreement

Параметры загрузки:

IP: 54.162.27.143

26 апреля 2023 г., 13:53:07

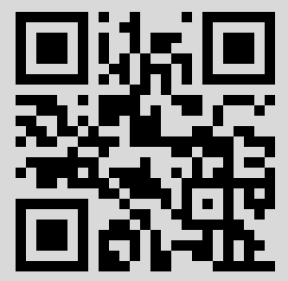




\section{ТЕОРЕМА О НУЛЯХ ЦЕЛЫХ ФУНКЦИЙ И ЕЕ ПРИМЕНЕНИЕ}

\section{В. П. Заставный}

Рассматриваются целые функции экспоненциального типа $\leqslant \sigma$, ограниченные и вещественные на $\mathbb{R}$, для которых вьполняется оценка $(-1)^{k} f(k \pi / \sigma+\tau) \geqslant 0, k \in \mathbb{Z}$. Доказано, что нули таких функций вещественные и простые за исключением, быть может, точек вида $k \pi / \sigma+\tau$, которые могут быть нулями кратности не более, чем 2 . Эти результаты применяются к конкретным классам функций и к вопросу устойчивости целых функций. Уточняются и дополняются некоторые результаты Пойа.

Библиография: 5 названий.

Введение. В работе Пойа $[1, \S 5]$ доказана следующая

ТЕОремА ГУРВИЦА. Пусть $g \in L(0, \sigma) u U(z):=\int_{0}^{1} g(t) \cos z t d t . \quad E c \Omega u(-1)^{k} \times$ $U(k \pi)>0$ для всех $k \in \mathbb{Z}$, то все нули функции $U$ вещественные и простые, причем они расположены по одному в каждом интервале $(k \pi,(k+1) \pi), k \in \mathbb{Z}$.

Доказательство этой теоремы основано на следующих фактах:

1) для функции $U$ справедлива формула $[1, \S 5,(38)]$

$$
\frac{U(z)}{\sin z}=\frac{U(0)}{z}+\sum_{k=1}^{+\infty}(-1)^{k} U(k \pi)\left[\frac{1}{z-k \pi}+\frac{1}{z+k \pi}\right]
$$

2) все нули рациональной дроби

$$
\sum_{k=-n}^{n} \frac{(-1)^{k} U(k \pi)}{z-k \pi}
$$

вешественные и простые, причем они расположены по одному в каждом интервале $(k \pi$, $(k+1) \pi), k=-n, \ldots, n-1, n \in \mathbb{N}$;

3) теорема о нулях предельной функции.

При доказательстве существенно используется то, что указанные в теореме неравенства строгие. В п. 1 данной работы теорема Гурвица распространена на целые функции $f \not \equiv 0$ экспоненциального типа $\leqslant \sigma$, которые вещественны и ограниченны на $\mathbb{R}$ и для которых при некоторых $n=n(f) \in\{0,1\}, \tau=\tau(f) \in \mathbb{R}$ и при всех $k \in \mathbb{Z}$ вьполняются неравенства $(-1)^{n+k} f(k \pi / \sigma+\tau) \geqslant 0$ (допускаются и нестрогие неравенства). В теореме 1 доказано, что нули таких функций вещественные и простые, за исключением, быть

Работа поддержана FFUY, проект № 01.07/00241. 
может, точек вида $k \pi / \sigma+\tau$, которые могут быть нулями кратности не более, чем 2, и в каждом интервале

$$
I_{p}:=\left(\frac{(p-1) \pi}{\sigma}+\tau, \frac{p \pi}{\sigma}+\tau\right), \quad p \in \mathbb{Z},
$$

может находиться лишь один нуль функции $f$. Доказано еще, что $\sup _{x \in \mathbb{R}} x^{2}|f(x)|=$ $+\infty$. Доказательство теоремы 1 основано на другой интерполящионной формуле (1) c использованием теоремы о промежуточных значениях.

В п. 2 теорема 1 применяется для изучения нулей функций

$$
f_{\alpha}(x):=\int_{0}^{\sigma} g(t) \sin (t x+\alpha) d t, \quad \text { где } g \in L(0, \sigma), \quad \sigma>0 .
$$

Ранее нули таких функций изучал Пойа [1] $(\sigma=1)$. Следуя ему, будем говорить, что функция $g$ является исключительной на $(0,1)$, если она кусочно постоянна, имеет конечное число точек разрыва и все ее точки разрьва являются рациональными числами. В противном случае будем говорить, что функция $g$ является на $(0,1)$ функиией общего вида. Пойа доказал следующие утверждения.

Теорема А. Пусть $g(t)>0$ и не убывает на $(0,1)$. Тогда выполнены следующие утверждения:

1) функиия $f_{\alpha}$ имеет только вещественные нули, а если дополнительно $g$ является функиией общего вида, то все нули $f_{\alpha}$ простые $[1, \S 3]$;

2) при $\alpha=\pi / 2$ функция $f_{\alpha}$ имеет только простые вещественные нули; кроме того, в интервале $(0, \pi / 2)$ нулей нет, а в каждом интервале $(\pi / 2+k \pi, 3 \pi / 2+k \pi)$, $k \in \mathbb{N} \cup\{0\}$, содержится только один нуль $[1, \S 6$, п. I];

3) если $g$ является функиией общего вида, то при $\alpha=0$ функиия $f_{\alpha}$ имеет только простые вещественные нули; кроме того, в интервале $(0, \pi)$ нулей нет, а в каждом интервале $(k \pi,(k+1) \pi), k \in \mathbb{N}$, содержится только один нуль $[1, \S 6$, п. II].

Доказательство утверждения 1) теоремы А основано на следующих фактах:

а) теорема о нулях полиномов $[1, \S 2]$

$$
\sum_{k=0}^{n} a_{k} \cos (k x+d), \quad 0<a_{0}<a_{1}<\cdots<a_{n}
$$

b) теорема о нулях предельной функции и теорема из $[1, \S 1]$, которая утверждает, что если $g(t)>0$, не убывает на $(0,1)$ и является функцией общего вида, то нули функции $\int_{0}^{1} g(t) e^{z t} d t$ лежат в полуплоскости $\operatorname{Re} z<0$.

Доказательство утверждений 2) и 3) теоремы А основано на таких фактах:

a) если $\alpha=\pi / 2$ или $\alpha=0$, то $(-1)^{k} f_{\alpha}(k \pi-\alpha)<0, k \in \mathbb{N}$, и для функции $f_{\alpha}$ справедлива формула $[1, \S 6,(43),(44)]$

$$
\frac{f_{\alpha}(z)}{z \sin (z+\alpha)}=\frac{f_{\alpha}(0)+f_{\alpha}^{\prime}(0)}{z}+\sum_{k=1}^{+\infty} \frac{(-1)^{k} f_{\alpha}(k \pi-\alpha)}{k \pi-\alpha}\left[\frac{1}{z-k \pi+\alpha}+\frac{1}{z+k \pi-\alpha}\right]
$$


b) теорема о нулях рациональной дроби $[1, \S 5$, п. IV]

$$
\frac{B}{z}+\sum_{k=1}^{n} A_{k}\left[\frac{1}{z-a_{k}}+\frac{1}{z+a_{k}}\right]
$$

где $0<a_{0}<a_{1}<\cdots<a_{n}, A_{k}>0,1 \leqslant k \leqslant n, B \in \mathbb{R}$,

c) теорема о нулях предельной функции.

В п. 2 данной работы доказана теорема 2, в которой теорема А распространена на неубывающие функции $g$ с условием $g(x) \geqslant 0$ и $g \not \equiv 0$ на $(0, \sigma)$ (строгоенеравенство заменено на нестрогое) и, кроме того, утверждения 2) и 3) распространены на любые значения $\alpha$. При этом роль исключительных функций $g$ вьполняют классы $M_{\alpha}(\sigma)$, которые описьваются явньп образом равенствами $(5),(7),(10),(12)$, что соответствует функциям $g$, для которых при некотором целом $k \neq 0$ справедливо равенство $f_{\alpha}((k \pi-\alpha) / \sigma)=0$. Отметим, что если $g(t)>0$ и не убьвает на $(0,1)$, то $g$ является исключительной в смысле Пойа тогда и только тогда, когда $g \in M_{0}(1)$. В случае ограниченной функции $g$ доказательство теоремы 2 основано на простом следствии 3 из теоремы 1 и на лемме 1 , в которой доказаны нестрогие неравенства

$$
\begin{aligned}
& (-1)^{k} f_{\alpha}\left(\frac{k \pi-\alpha}{\sigma}\right) \leqslant 0, \quad k \geqslant 1, \\
& (-1)^{k} f_{\alpha}\left(\frac{k \pi-\alpha}{\sigma}\right) \geqslant 0, \quad k \leqslant 0, \quad k \in \mathbb{Z},
\end{aligned}
$$

а общий случай получается из этого предельным переходом.

Для вьпуклых вниз функций $g$ Пойа доказал утверждения, которые уточняют расположение нулей $f_{\alpha}$.

ТЕОремА В. Пусть функиия $g$ строго возрастает и выпукла вниз на $(0,1)$. Tогда

1) если $g(+0)=0$, то при $\alpha=0$ функиия $f_{\alpha}$ имеет только простые вещественные нули и в кажсдом интервале $(k \pi, \pi / 2+k \pi), k \in \mathbb{N}$, содержится только один нуль $[1, \S 6$, п. III];

2) если $g(t)>0^{1}$ на $(0,1)$ и правая производная $g^{\prime}(t)$ является функиией общего вида, то при $\alpha=\pi / 2$ у функиии $f_{\alpha}$ в каждом интервале $(\pi / 2+k \pi,(k+1) \pi)$, $k \in \mathbb{N} \cup\{0\}$, содерэится только один нуль $[1, \S 6$, п. IV];

3) если $g(a)=0$ при некотором $a \in(0,1)$, то при $\alpha=\pi / 2$ функиия $f_{\alpha}$ имеет только простые нули, которые все вещественные (если $\left.f_{\alpha}(0)>0\right)$, или среди ее нулей ровно два чисто мнимые, а остальные вещественные $\left(\right.$ если $\left.f_{\alpha}(0)<0\right)[1$, $\S 6$, п. VI].

Доказательство утверждений 1), 2) теоремы В следующее: если $\alpha=0$ или $\alpha=\pi / 2$, то

$$
(-1)^{k} f_{\alpha}\left(k \pi+\frac{\pi}{2}-\alpha\right)>0, \quad k \in \mathbb{N},
$$

\footnotetext{
${ }^{1} Э$ то условие у Пойа пропущено, хотя при доказательстве оно неявно используется (используется неравенство $[1,(43)]$, доказанное при условии $g(t)>0)$.
} 
и дальшенадо воспользоваться теоремой А. Доказательство утверждения 3) теоремы В аналогично доказательству утверждения 2) теоремы А. В конце п. 2 доказано следствие 4 , в котором утверждение 1 ) теоремы В распространено на любые значения $\alpha$. Доказано также следствие 5 , из которого получаются утверждения 2) и 3 ) теоремы В. При этом в следствиях 4 и 5 условие " $g$ строго возрастает" заменено на условие " $g$ не убьвает и $g \not \equiv 0$ на $(0, \sigma)$ ", а следствие 5 доказано без предположения, что $g^{\prime}$ является функцией общего вида. Следствия 4 и 5 вытекают из теорем 1,2 и леммы 2 , в которой доказаны нестрогие неравенства

$$
(-1)^{k} h_{\alpha}\left(\frac{k \pi-\alpha}{\sigma}\right) \geqslant 0, \quad k \in \mathbb{Z},
$$

(здесь $\left.h_{\alpha} \equiv f_{\alpha+\pi / 2}\right)$ и найдены условия, при которых возможно равенство. Отметим еще, что из следствия 5 легко получается утверждение $[1, \S 6$, п. V], в котором речь идет о нулях $f_{\alpha}$ при $\alpha=\pi / 2$, когда функция $g(t)>0$, строго убьвает и выпукла вверх.

В п. 3 изучаются нули функции

$$
F(z):=\int_{0}^{\sigma} g(t) e^{z t} d t, \quad g \in L(0, \sigma) .
$$

Пойа доказал следующую теорему $[1, \S 1](\sigma=1)$.

Tеорема С. Пусть $g(t)>0$ ине убьвает на $(0,1)$. Тогда

1) если $g$ является функиией общего вида, то все нули функиии $F$ лежат в полуплоскости $\operatorname{Re} z<0$;

2) если $g$ является исключительной, то все нули функиии $F$ лежат в полуплоскости $\operatorname{Re} z \leqslant 0$ и на прямой $\operatorname{Re} z=0$ леэит бесконечно много ее нулей.

В утверждении 1 ) теоремы $\mathrm{C}$ речь идет о том, что целая функция $F$ является устойчивой, т.е. не имеет нулей в замкнутой полуплоскости $\operatorname{Re} z \geqslant 0$. В данной работе доказана теорема 3 , в которой теорема С распространена на неубывающие функции $g$ с условием $g(t) \geqslant 0$ и $g \not \equiv 0$ на $(0, \sigma)$. При этом роль исключительных функций вьполняет класс $\bigcup_{0 \leqslant \alpha<\pi} M_{\alpha}(\sigma)$. В первом случае доказано более сильное утверждение: $F$ является сильно устойчивой (определение см. в п. 3). Доказательство теоремы 3 , в отличие от доказательства Пойа, основано на критерии Чеботарёва для сильно устойчивых функций с использованием теоремы 2 . Из теоремы 3 в качестве простого следствия 6 получается утверждение о положительной определенности специального класса функций.

В заключение отметим, что использование результатов теоремы А можно найти в [2].

1. Обозначим через $B_{\sigma}, \sigma>0$, класс целых функций экспоненциального типа $\leqslant \sigma$, ограниченных на вещественной оси (класс Бернштейна).

ТЕОРема 1. Пусть выполнены условия

1) $f \in B_{\sigma}$, вещественна на вещественной оси $u f \not \equiv 0$;

2) при некоторых $n=n(f) \in\{0,1\}, \tau=\tau(f) \in \mathbb{R} u$ при всех $k \in \mathbb{Z}$ выполняются неравенства

$$
(-1)^{n+k} f\left(\frac{k \pi}{\sigma}+\tau\right) \geqslant 0 .
$$

Тогда имеют место следующие утверждения. 
1) В каждом интервале

$$
I_{p}:=\left(\frac{(p-1) \pi}{\sigma}+\tau, \frac{p \pi}{\sigma}+\tau\right), \quad p \in \mathbb{Z},
$$

мохсет находиться лишь один нуль функиии $f$, и если он есть, то он простой.

2) Выполнено равенство

$$
\sup _{x \in \mathbb{R}} x^{2}|f(x)|=+\infty
$$

3) Все нули функции $f$ вещественные.

4) Если при некотором $p \in \mathbb{Z}$ число $z_{p}:=p \pi / \sigma+\tau$ является нулем функиии $f$, то его кратность не превышает 2 и в одном из интервалов $I_{p}$ и $I_{p+1}$ нулей функиии $f$ нет. Если число $z_{p}$ является нулем кратности 2, то $(-1)^{n+p} f^{(2)}\left(z_{p}\right)<0$ $u(-1)^{n+p} f(x)<0$ при $x \in I_{p} \cup I_{p+1}$, а числа $z_{p-1}$ и $z_{p+1}$ могут бъть только простыми нулями.

Класс функций, удовлетворяющих условиям теоремы 1 , обозначим через $B_{\sigma}(\tau)$.

ДокАЗАТЕЛЬСТво. 1) Для функций $f \in B_{\sigma}$ справедлива следующая интерполяционная формула (см. [3, с. 188]):

$$
\sin \alpha f^{\prime}(x)-\sigma \cos \alpha f(x)=\sigma \sum_{k=-\infty}^{\infty}(-1)^{k-1} \frac{\sin ^{2} \alpha}{(\alpha-k \pi)^{2}} f\left(\frac{k \pi-\alpha}{\sigma}+x\right), \quad x, \alpha \in \mathbb{R}
$$

Полагая $\alpha=\sigma(x-\tau)$, получаем

$\sin \sigma(x-\tau) f^{\prime}(x)-\sigma \cos \sigma(x-\tau) f(x)=\sigma \sum_{k=-\infty}^{\infty}(-1)^{k-1} \frac{\sin ^{2} \sigma(x-\tau)}{(\sigma(x-\tau)-k \pi)^{2}} f\left(\frac{k \pi}{\sigma}+\tau\right)$.

Учитьвая условие 2 ) теоремы, получаем, что при любом $x \in \mathbb{R}$ справедливо равенство

$\sin \sigma(x-\tau) f^{\prime}(x)-\sigma \cos \sigma(x-\tau) f(x)=(-1)^{n-1} \sigma \sum_{k=-\infty}^{\infty} \frac{\sin ^{2} \sigma(x-\tau)}{(\sigma(x-\tau)-k \pi)^{2}}\left|f\left(\frac{k \pi}{\sigma}+\tau\right)\right|$.

Предположим, что при некотором $p \in \mathbb{Z}$ в интервале $I_{p}$ есть нуль функции $f$. В этом случае $f(k \pi / \sigma+\tau) \neq 0$ при некотором $k \in \mathbb{Z}$ (в противном случае из равенства (1) вытекает, что $f(x)=c \sin \sigma(x-\tau), c \neq 0$, но у этой функции все нули в точках $k \pi / \sigma+\tau)$. Если $x_{0} \in I_{p}$ и $f\left(x_{0}\right)=0$, то из равенства $(2)$ следует, что $(-1)^{n+p} f^{\prime}\left(x_{0}\right)>0$. Предположим, что в интервале $I_{p}$ число нулей функции $f$ более одного. Так как $f$ целая и $f \not \equiv 0$, то в этом интервале лишь конечное число нулей. Возьмем любые два соседние $x_{1}$ и $x_{2}$, т.е. $x_{1}<x_{2}$ и $f(x) \neq 0$ при $x \in\left(x_{1}, x_{2}\right)$. Считая для определенности $n+p$ четным (и значит $f^{\prime}\left(x_{i}\right)>0$ ), заключаем, что

$$
\exists \xi_{1}, \xi_{2}: \quad x_{1}<\xi_{1}<\xi_{2}<x_{2}, \quad f\left(\xi_{1}\right)>0, \quad f\left(\xi_{2}\right)<0 .
$$

Поэтому между $\xi_{1}$ и $\xi_{2}$ имеется еще хотя бы один нуль функции $f$. Полученное противоречие доказьвает первое утверждение теоремы 1. 
2) Предположим, что $\sup _{\mathbb{R}} x^{2}|f(x)|<+\infty$. Тогда функция

$$
g(z):=f(z)\left(z-x_{0}\right)^{2}, \quad \text { где } \quad x_{0} \in\left(\tau, \frac{\pi}{\sigma}+\tau\right)
$$

принадлежит классу $B_{\sigma}(\tau)$ и число $x_{0}$ является нулем кратности не менее, чем 2 . Это противоречит первому утверждению.

3) Предположим, что $f\left(z_{0}\right)=0$ при некотором $z_{0} \in \mathbb{C} \backslash \mathbb{R}$. Тогда $f\left(\bar{z}_{0}\right)=0$ и, значит, функция

$$
g(z):=\frac{f(z)}{\left(z-z_{0}\right)\left(z-\bar{z}_{0}\right)}
$$

принадлежит классу $B_{\sigma}(\tau)$. Очевидно $\sup _{\mathbb{R}} x^{2}|g(x)|<+\infty$, что противоречит второму утверждению.

4) Пусть при некотором $p \in \mathbb{Z}$ число $z_{p}:=p \pi / \sigma+\tau$ является нулем функции $f$. Далее доказательство разобьем на несколько подпунктов.

а) Если $f^{\prime}\left(z_{p}\right)=0$ и $(-1)^{n+p} f^{\prime \prime}\left(z_{p}\right) \geqslant 0$, то функция

$$
g(z):=\frac{f(z)}{\left(z-z_{p}\right)^{2}}
$$

принадлежит классу $B_{\sigma}(\tau)$ и $\sup _{\mathbb{R}} x^{2}|g(x)|<+\infty$, что противоречит второму утверждению. Следовательно, $(-1)^{n+p} f^{\prime \prime}\left(z_{p}\right)<0$.

b) Если в обоих интервалах $I_{p}$ и $I_{p+1}$ имеются нули функции $f$, например $x_{1} \in I_{p}$ и $x_{2} \in I_{p+1}$, то функция

$$
g(z):=\frac{f(z)\left(z-z_{p}\right)^{2}}{\left(z-x_{1}\right)\left(z-x_{2}\right)}
$$

принадлежит классу $B_{\sigma}(\tau)$ и число $z_{p}$ является нулем кратности более, чем 2 , что противоречит пункту а).

c) Пусть $z_{p}$ является нулем функции $f$ кратности 2. Предположим, например, что число $x_{1} \in I_{p}$ является нулем функции $f$. Тогда функция

$$
g(z):=\frac{f(z)}{\left(z-x_{1}\right)\left(z-z_{p}\right)}
$$

принадлежит классу $B_{\sigma}(\tau)$ и $\sup _{\mathbb{R}} x^{2}|g(x)|<+\infty$, что противоречит второму утверждению. Поэтому в интервале $I_{p}$ нулей функции $f$ нет. Аналогично и в интервале $I_{p+1}$ нет нулей. По доказанному в пункте а) $(-1)^{n+p} f^{\prime \prime}\left(z_{p}\right)<0$ и, значит, $(-1)^{n+p} f(x)<0$ при $x \in I_{p} \cup I_{p+1}$.

d) Предположим, что $z_{p}$ и $z_{p-1}$ являются нулями функции $f$ кратности 2 . Тогда функция

$$
g(z):=\frac{f(z)}{\left(z-z_{p-1}\right)\left(z-z_{p}\right)}
$$

принадлежит классу $B_{\sigma}(\tau)$ и $\sup _{\mathbb{R}} x^{2}|g(x)|<+\infty$, что противоречит второму утверждению. 
СлЕДСТВИЕ 1. Пусть $f \in B_{\sigma}(\tau)$. Тогда для всех $m \in \mathbb{N}$ выполнено равенство $\sup _{\mathbb{R}} x^{2}\left|f^{(m)}(x)\right|=+\infty$ и все нули функиии $f^{(m)}$ вещественные и простые. Если дополнительно $f(z) \not \equiv c \sin \sigma(z-\tau)$, то нули $f^{(m)}$ располагаются по одному в каждом интервале

$$
\left(\frac{(p-1) \pi}{\sigma}+\frac{m \pi}{2 \sigma}+\tau, \frac{p \pi}{\sigma}+\frac{m \pi}{2 \sigma}+\tau\right), \quad p \in \mathbb{Z} .
$$

ДокАЗАТЕльство. Если $f(z) \equiv c \sin \sigma(z-\tau), c \neq 0$, то утверждение очевидно. Поэтому считаем, что $f(z) \not \equiv c \sin \sigma(z-\tau)$. В этом случае $f(k \pi / \sigma+\tau) \neq 0$ при некотором $k \in \mathbb{Z}$. Подставляя в $(2) x=p \pi / \sigma+\pi /(2 \sigma)+\tau$, получаем, что

$$
(-1)^{n-1+p} f^{\prime}\left(\frac{p \pi}{\sigma}+\frac{\pi}{2 \sigma}+\tau\right)>0 \quad \forall p \in \mathbb{Z} .
$$

Значит, $f^{\prime} \in B_{\sigma}(\pi /(2 \sigma)+\tau)$, и осталось применить теорему 1 . При $m=1$ следствие 1 доказано, а при остальных $m$ доказательство проводится индукцией.

СлЕДСТВИЕ 2. Пусть функиия $f \in B_{\sigma}$, принимает на вещественной оси вещественные значения, является четной, $f(0)>0 u(-1)^{k+1} f(k \pi / \sigma) \geqslant 0$ для всех $k \in \mathbb{N}$. Тогда выполнены следующие утверждения.

1) Имеет место оценка

$$
f(x)>f(0) \frac{\sin \sigma x}{\sigma x} \quad n p u \quad x \in\left(-\frac{\pi}{\sigma}, 0\right) \cup\left(0, \frac{\pi}{\sigma}\right)
$$

$u f^{\prime \prime}(0)>-f(0) \sigma^{2} / 3$.

2) В каждом интервале $((p-1) \pi / \sigma, p \pi / \sigma), p \in \mathbb{Z} \backslash\{0,1\}$, может находиться лишь один нуль функиии $f$, и если он есть, то он простой. Если число $р \pi / \sigma$, $p \in \mathbb{Z} \backslash\{0\}$, является нулем функиии $f$, то его кратность не превышает 2.

3) Если $f$ имеет комплекснье нули, то их число с учетом кратности равно 2 и они чисто мнимые.

ДокАЗАТЕЛЬСтво. 1) Рассмотрим функцию

$$
g(z):=f(z)-f(0) \frac{\sin \sigma z}{\sigma z}
$$

Очевидно, что $g(0)=g^{\prime}(0)=0$ и $g \in B_{\sigma}(0)$. По теореме 1 (утверждение 4) $g(x)>0$ при $x \in(-\pi / \sigma, 0) \cup(0, \pi / \sigma)$ и $g^{\prime \prime}(0)>0$. Поэтому

$$
f(x)>f(0) \frac{\sin \sigma x}{\sigma x} \quad \text { при } \quad x \in\left(-\frac{\pi}{\sigma}, 0\right) \cup\left(0, \frac{\pi}{\sigma}\right)
$$

и $f^{\prime \prime}(0)>-f(0) \sigma^{2} / 3$.

2) Предположим, что в некотором интервале $((p-1) \pi / \sigma, p \pi / \sigma)$ число нулей функции $f$ с учетом кратности более одного, например, $x_{1} \leqslant x_{2}$. Тогда функция

$$
g(z):=\frac{f(z) z^{2}}{\left(z^{2}-x_{1}^{2}\right)\left(z^{2}-x_{2}^{2}\right)}
$$


принадлежит классу $B_{\sigma}(0) \sup _{x \in \mathbb{R}} x^{2}|g(x)|<+\infty$, что противоречит утверждению 2) теоремы 1. Если число $p \pi / \sigma, p \neq 0$, является нулем функции $f$ кратности вьше 2 , то надо рассмотреть функцию $f(z) z^{2} /\left(\sigma^{2} z^{2}-p^{2} \pi^{2}\right)^{2}$.

3) Предположим, что число $z_{0}=\alpha+i \beta$, где $\alpha, \beta \in \mathbb{R}, \beta \neq 0$, является нулем функции $f$. Если $\alpha \neq 0$, то функция

$$
g(z):=\frac{f(z) z^{2}}{\left(z^{2}-z_{0}^{2}\right)\left(z^{2}-\bar{z}_{0}^{2}\right)}
$$

принадлежит классу $B_{\sigma}(0)$, и $\sup _{x \in \mathbb{R}} x^{2}|g(x)|<+\infty$, что противоречит утверждению 2) теоремы 1 . Поэтому $\alpha=0$. Тогда функция $f(z) z^{2} /\left(z^{2}+\beta^{2}\right)$ принадлежит классу $B_{\sigma}(0)$, и по теореме 1 у нее все нули вещественные.

СлЕДСТВИЕ 3. Пусть выполнены условия:

1) $f \in B_{\sigma}$, вещ,ественна на вещественной оси $и f \not \equiv 0$;

2) при некоторых $n \in\{0,1\}, \tau \in \mathbb{R} u$ при всех $k \in \mathbb{Z}$ выполняются неравенства

$$
(-1)^{n+k} f\left(\frac{k \pi}{\sigma}+\tau\right) \leqslant 0, \quad k \geqslant 1, \quad(-1)^{n+k} f\left(\frac{k \pi}{\sigma}+\tau\right) \geqslant 0, \quad k \leqslant 0
$$

3) $\sup _{x \in \mathbb{R}}|x f(x)|<+\infty$.

Тогда выполнены следующие утверждения.

1) Все нули функиии $f$ вещественные. В интервале

$$
I_{p}:=\left(\frac{(p-1) \pi}{\sigma}+\tau, \frac{p \pi}{\sigma}+\tau\right), \quad p \in \mathbb{Z} \backslash\{1\},
$$

может находиться лишь один нуль функиии $f$, и если он есть, то он простой. Кроме того, $(-1)^{n} f(x)>0$ при $x \in I_{1}:=(\tau, \pi / \sigma+\tau)$.

2) Если при некотором $k \in\{0,1\}$ число $k \pi / \sigma+\tau$ является нулем функиии $f$, то его кратность равна 1, а в смехных с ним интервалах $I_{k}$ и $I_{k+1}$ нулей функиии $f$ нem.

ДокАЗАТЕЛЬСТво. Пусть $x_{0} \in(\tau, \pi / \sigma+\tau)$. Тогда $g(z):=\left(z-x_{0}\right) f(z) \in B_{\sigma}(\tau)$, и к ней можно применить теорему 1 . Значит, в интервале $I_{1}$ функция $g$ имеет единственньй нуль $x_{0}$ и $(-1)^{n} g^{\prime}\left(x_{0}\right)>0$. Поэтому $f(x) \neq 0$ при $x \in I_{1}$, а так как еще $g^{\prime}\left(x_{0}\right)=$ $f\left(x_{0}\right)$, то $(-1)^{n} f(x)>0$ при $x \in I_{1}$. Утверждение 1) доказано. Для доказательства утверждения 2) надо в качестве $x_{0}$ взять $k \pi / \sigma+\tau, k \in\{0,1\}$, и к $g \in B_{\sigma}(\tau)$ применить утверждение 4) в теореме 1.

2. Начнем с предварительного утверждения.

ЛЕмма 1. Пусть функиия $g \in L(0, \sigma), g(x) \geqslant 0$ и не убывает на $(0, \sigma), 0 \leqslant$ $\alpha<\pi, a$

$$
f_{\alpha}(x):=\int_{0}^{\sigma} g(t) \sin (t x+\alpha) d t .
$$

Тогда для всех $k \in \mathbb{Z}$ выполняются неравенства

$$
(-1)^{k} f_{\alpha}\left(\frac{k \pi-\alpha}{\sigma}\right) \leqslant 0, \quad k \geqslant 1, \quad(-1)^{k} f_{\alpha}\left(\frac{k \pi-\alpha}{\sigma}\right) \geqslant 0, \quad k \leqslant 0 .
$$


При этом равенство

$$
f_{\alpha}\left(\frac{k \pi-\alpha}{\sigma}\right)=0, \quad k \in \mathbb{Z} \backslash\{0\}
$$

возможно тогда и только тогда, когда g представима одной из формул (5), (7), $(10),(12)$ (см. нижее), и в этом случае, если $g(x) \not \equiv 0$ на $(0, \sigma)$, то число $(k \pi-\alpha) / \sigma$ является нулем функиии $f_{\alpha}$ кратности 2.

ДОКАЗАТЕЛЬСТВо. Очевидно, что

$$
f_{\alpha}\left(-\frac{\alpha}{\sigma}\right)=\int_{0}^{\sigma} g(t) \sin \left(\frac{\alpha(\sigma-t)}{\sigma}\right) d t \geqslant 0
$$

и $f_{\alpha}(-\alpha / \sigma)=0$ тогда и только тогда, когда либо $\alpha=0$, либо $g(t) \equiv 0$ на $(0, \sigma)$.

Пусть $k \in \mathbb{N}$. Тогда

$$
f_{\alpha}\left(\frac{k \pi-\alpha}{\sigma}\right)=\frac{\sigma}{k \pi-\alpha} \int_{\alpha}^{k \pi} g\left(\frac{\sigma(u-\alpha)}{k \pi-\alpha}\right) \sin u d u
$$

Если $k$ нечетное, то отрезок интегрирования в (4) разобьем на отрезки $[\alpha, \pi],[\pi, 3 \pi], \ldots$, $[k \pi-2 \pi, k \pi]$. Интеграл по каждому из этих отрезков неотрицателен, поэтому и выражение (4) неотрищательно. Если при этом $f_{\alpha}((k \pi-\alpha) / \sigma)=0$, то очевидно

$$
g(x) \equiv c_{p}, \quad x \in\left(x_{p-1}, x_{p}\right), \quad 0 \leqslant p \leqslant \frac{k-1}{2}
$$

где

$$
x_{-1}=0, \quad x_{p}=\sigma \frac{(2 p+1) \pi-\alpha}{k \pi-\alpha}, \quad 0 \leqslant p \leqslant \frac{k-1}{2}, \quad 0=c_{0} \leqslant c_{1} \leqslant \cdots \leqslant c_{(k-1) / 2} .
$$

В этом случае

$$
f_{\alpha}(x)=\frac{2}{x} \sin \left(\frac{\sigma \pi x}{k \pi-\alpha}\right) \sum_{p=0}^{(k-1) / 2} c_{p} \sin \left(\frac{2 p \pi-\alpha}{k \pi-\alpha} \sigma x+\alpha\right)
$$

Из $(5),(6)$ видно, что если не все $c_{p}$ равны нулю, то $k \neq 1$ и число $(k \pi-\alpha) / \sigma$ является нулем функции $f_{\alpha}$ кратности 2 .

Если $k$ четное, то отрезок интегрирования в (4) разобьем на отрезки $[\alpha, 2 \pi],[2 \pi, 4 \pi]$, $\ldots,[k \pi-2 \pi, k \pi]$. Интеграл по каждому из этих отрезков неположителен, поэтому и выражение (4) неположительно. Если при этом $f_{\alpha}((k \pi-\alpha) / \sigma)=0$, то очевидно

$$
g(x) \equiv c_{p}, \quad x \in\left(x_{p-1}, x_{p}\right), \quad 0 \leqslant p \leqslant \frac{k-2}{2}
$$

где

$$
x_{-1}=0, \quad x_{p}=\sigma \frac{(2 p+2) \pi-\alpha}{k \pi-\alpha}, \quad 0 \leqslant p \leqslant \frac{k-2}{2}, \quad 0 \leqslant c_{0} \leqslant c_{1} \leqslant \cdots \leqslant c_{(k-2) / 2},
$$


и если $0<\alpha<\pi$, то $c_{0}=0$. В этом случае

$$
f_{\alpha}(x)=\frac{2}{x} \sin \left(\frac{\sigma \pi x}{k \pi-\alpha}\right) \sum_{p=0}^{(k-2) / 2} c_{p} \sin \left(\frac{(2 p+1) \pi-\alpha}{k \pi-\alpha} \sigma x+\alpha\right) .
$$

Из (7), (8) видно, что если не все $c_{p}$ равны нулю, то $k \neq 2$ при $0<\alpha<\pi$ и число $(k \pi-\alpha) / \sigma$ является нулем функции $f_{\alpha}$ кратности 2 .

Рассмотрим теперь случай, когда $k \in \mathbb{Z}$ и $k \leqslant-1$. В этом случае

$$
f_{\alpha}\left(\frac{k \pi-\alpha}{\sigma}\right)=\frac{\sigma}{k \pi-\alpha} \int_{-\alpha}^{-k \pi} g\left(\frac{\sigma(u+\alpha)}{\alpha-k \pi}\right) \sin u d u .
$$

Если $k$ нечетное, то отрезок интегрирования в (9) разобьем на отрезки $[-\alpha, \pi],[\pi, 3 \pi]$, $\ldots,[-k \pi-2 \pi,-k \pi]$. Интеграл по каждому из этих отрезков неотрицателен, поэтому выражение (9) неположительно. Если при этом $f_{\alpha}((k \pi-\alpha) / \sigma)=0$, то очевидно

$$
g(x) \equiv c_{p}, \quad x \in\left(x_{p-1}, x_{p}\right), \quad 0 \leqslant p \leqslant \frac{-k-1}{2},
$$

где

$$
x_{-1}=0, \quad x_{p}=\sigma \frac{(2 p+1) \pi+\alpha}{\alpha-k \pi}, \quad 0 \leqslant p \leqslant \frac{-k-1}{2}, \quad 0=c_{0} \leqslant c_{1} \leqslant \cdots \leqslant c_{(-k-1) / 2} .
$$

В этом случае

$$
f_{\alpha}(x)=\frac{2}{x} \sin \left(\frac{\sigma \pi x}{\alpha-k \pi}\right) \sum_{p=0}^{(-k-1) / 2} c_{p} \sin \left(\frac{2 p \pi+\alpha}{\alpha-k \pi} \sigma x+\alpha\right) .
$$

Из $(10),(11)$ видно, что если не все $c_{p}$ равны нулю, то $k \neq-1$ и число $(k \pi-\alpha) / \sigma$ является нулем функции $f_{\alpha}$ кратности 2 .

Если $k$ четное, то отрезок интегрирования в (9) разобьем на отрезки $[-\alpha, 0],[0,2 \pi]$, $\ldots,[-k \pi-2 \pi,-k \pi]$. Интеграл по каждому из этих отрезков неположителен, поэтому выражение (9) неотрищательно. Если при этом $f_{\alpha}((k \pi-\alpha) / \sigma)=0$, то очевидно

$$
g(x) \equiv c_{p}, \quad x \in\left(x_{p-1}, x_{p}\right), \quad 0 \leqslant p \leqslant-\frac{k}{2},
$$

где

$$
x_{-1}=0, \quad x_{p}=\sigma \frac{2 p \pi+\alpha}{\alpha-k \pi}, \quad 0 \leqslant p \leqslant-\frac{k}{2}, \quad 0=c_{0} \leqslant c_{1} \leqslant \cdots \leqslant c_{-k / 2} .
$$

В этом случае

$$
f_{\alpha}(x)=\frac{2}{x} \sin \left(\frac{\sigma \pi x}{\alpha-k \pi}\right) \sum_{p=0}^{-k / 2} c_{p} \sin \left(\frac{(2 p-1) \pi+\alpha}{\alpha-k \pi} \sigma x+\alpha\right) .
$$

Из равенства (13) видно, что если не все $c_{p}$ равны нулю, то число $(k \pi-\alpha) / \sigma$ является нулем функции $f_{\alpha}$ кратности 2 .

Будем говорить, что функция $g$ принадлежит классу $M_{\alpha}(\sigma), 0 \leqslant \alpha<\pi, \sigma>0$, если она представляется одной из формул $(5),(7),(10),(12)$. 
Теорема 2. Пусть функиия $g \in L(0, \sigma), g(x) \geqslant 0, g(x) \not \equiv 0$ и не убъвает на $(0, \sigma), \quad 0 \leqslant \alpha<\pi, a$

$$
f_{\alpha}(x):=\int_{0}^{\sigma} g(t) \sin (t x+\alpha) d t .
$$

Тогда выполнены следующие утверждения.

1) Если $g \in M_{\alpha}(\sigma)$, то все нули функиии $f_{\alpha}$ вещественные и в каждом интервале

$$
\left(\frac{(p-1)-\alpha \pi}{\sigma}, \frac{p \pi-\alpha}{\sigma}\right), \quad p \in \mathbb{Z} \backslash\{1\},
$$

может находиться лишь один нуль, и если он есть, то он простой, а в интервале $(-\alpha / \sigma,(\pi-\alpha) / \sigma)$ нулей нет. Кроме того:

а) среди чисел $(k \pi-\alpha) / \sigma, k \in \mathbb{Z}$, имеются нули $f_{\alpha}$;

б) если $0<\alpha<\pi$, то число $(k \pi-\alpha) / \sigma$ при $k \in\{ \pm 1,0,2\}$ не является нулем $f_{\alpha}$, а при $k \in \mathbb{Z} \backslash\{ \pm 1,0,2\}$, если это число и является нулем, то его кратность равна 2 ;

в) если $\alpha=0$, то число $k \pi / \sigma$ при $k= \pm 1$ не является нулем $f_{0}$, при $k=0$ это число является простым нулем, а при $k \in \mathbb{Z} \backslash\{ \pm 1,0\}$, если это число $u$ является нулем $f_{0}$, то его кратность равна 2.

2) Если $g \notin M_{\alpha}(\sigma)$, то у функиии $f_{\alpha}$ все нули вещ,ственные, в каждом интервале

$$
\left(\frac{(p-1) \pi-\alpha}{\sigma}, \frac{p \pi-\alpha}{\sigma}\right), \quad p \in \mathbb{Z} \backslash\{0,1\},
$$

и в полуинтервале $(-\pi / \sigma-\alpha / \sigma,-\alpha / \sigma]$ находится ровно один нуль и он простой, а в интервале $(-\alpha / \sigma, \pi / \sigma-\alpha / \sigma)$ нулей нет. При этом число $(k \pi-\alpha) / \sigma, k \in \mathbb{Z}$, является нулем только в случае $k=0, \quad \alpha=0$.

ДокАЗАтЕльство. 1) Если $g \in M_{\alpha}(\sigma)$, то $f_{\alpha}$ удовлетворяет условиям следствия 3 при $n=0, \tau=-\alpha / \sigma$ и осталось учесть результаты, полученные при доказательстве леммы 1.

2) В этом случае неравенства (3) при $k \neq 0$ строгие, а если $0<\alpha<\pi$, то они строгие и при $k=0$. Утверждение 2$)$ сразу получается, если $g$ ограничена на $(0, \sigma)$. В этом случае

$$
\sup _{x \in \mathbb{R}}\left|x f_{\alpha}(x)\right| \leqslant 2 \lim _{x \rightarrow \sigma-0} g(x)<+\infty
$$

и можно применить следствие 3 . Общий случай следует из этого предельным переходом. Пусть

$$
g_{n}(t):= \begin{cases}g(t), & 0 \leqslant t \leqslant \sigma-\frac{1}{n}, \\ g\left(\sigma-\frac{1}{n}\right)+t-\sigma+\frac{1}{n}, & \sigma-\frac{1}{n} \leqslant t \leqslant \sigma, \quad n>\sigma^{-1} .\end{cases}
$$

Функции $g_{n} \notin M_{\alpha}(\sigma)$ удовлетворяют условиям теоремы 2 и ограничены на $(0, \sigma)$. Значит, утверждение 2) теоремы 2 справедливо для соответствующих функций $f_{\alpha, n}$. Так как

$$
\int_{0}^{\sigma}\left|g(t)-g_{n}(t)\right| d t=\int_{\sigma-1 / n}^{\sigma}\left|g(t)-g_{n}(t)\right| d t \leqslant \int_{\sigma-1 / n}^{\sigma}|g(t)| d t+\frac{1}{2 n^{2}} \rightarrow 0, \quad n \rightarrow \infty
$$


то последовательность $f_{\alpha, n}$ стремится равномерно на каждом компакте из $\mathbb{C}$ к $f_{\alpha} \not \equiv 0$ и поэтому все нули функции $f_{\alpha}$ вещественные. Рассмотрим сначала случай, когда $0<$ $\alpha<\pi$. Тогда на границе прямоугольника

$$
K_{p}:=\left\{z \in \mathbb{C}:|\operatorname{Im} z| \leqslant 1, \frac{(p-1) \pi}{\sigma}-\frac{\alpha}{\sigma} \leqslant \operatorname{Re} z \leqslant \frac{p \pi}{\sigma}-\frac{\alpha}{\sigma}\right\}, \quad p \in \mathbb{Z},
$$

функция $f_{\alpha}$ не обращается в нуль и, значит, для всех $n \geqslant n(p)$ вьполняется неравенство

$$
\left|f_{\alpha, n}(z)-f_{\alpha}(z)\right|<\min _{u \in \partial K_{p}}\left|f_{\alpha}(u)\right| \leqslant\left|f_{\alpha}(z)\right| \quad \forall z \in \partial K_{p} .
$$

По теореме Руше внутри $K_{p}, p \in \mathbb{Z}$, функции $f_{\alpha}$ и $f_{\alpha, n}$ при $n \geqslant n(p)$ имеют одинаковое число нулей.

В случае $\alpha=0$ рассуждения остаются в силе, если вместо двух прямоугольников $K_{0}$ и $K_{1}$ рассмотреть один $K:=K_{0} \cup K_{1}$.

Лемма 2. Пусть функиия $g \in L(0, \sigma)$, не убывает и выпукла вниз на $(0, \sigma)$, $g(+0)=0, \quad 0 \leqslant \alpha<\pi, a$

$$
h_{\alpha}(x):=\int_{0}^{\sigma} g(t) \cos (t x+\alpha) d t .
$$

Тогда для всех $k \in \mathbb{Z}$ выполняются неравенства

$$
(-1)^{k} h_{\alpha}\left(\frac{k \pi-\alpha}{\sigma}\right) \geqslant 0 .
$$

Если при некотором $k \in \mathbb{Z}$ имеет место равенство $h_{\alpha}((k \pi-\alpha) / \sigma)=0$, mо $g$ представима в виде

$$
g(t)=\int_{0}^{t} g_{1}(u) d u, \quad g_{1} \in M_{\alpha}(\sigma)
$$

ДокаЗАТЕЛЬСтво. Пусть $k \in \mathbb{N}$ и $g(t):=0$ при $t \in[-\sigma \alpha /(k \pi-\alpha), 0]$. Тогда функция $\psi(u):=g(\sigma(u-\alpha) /(k \pi-\alpha))$ не убывает и выпукла вниз на $[0, k \pi)$ и

$$
h_{\alpha}\left(\frac{k \pi-\alpha}{\sigma}\right)=\frac{\sigma}{k \pi-\alpha} \int_{0}^{k \pi} \psi(u) \cos u d u .
$$

Если $k$ нечетное, то отрезок интегрирования в (16) разобьем на отрезки $[0, \pi],[\pi, 3 \pi]$, $\ldots,[k \pi-2 \pi, k \pi]$. Интеграл по каждому из этих отрезков неположителен, поэтому и выражение (16) неположительно. Если при этом $h_{\alpha}((k \pi-\alpha) / \sigma)=0$, то функция $\psi$ равна нулю на $[0, \pi]$ и линейна на остальных отрезках, а значит, $g$ представима в виде $(15)$, где $g_{1}$ имеет вид (5).

Если $k$ четное, то отрезок интегрирования в (16) разобьем на отрезки $[0,2 \pi],[2 \pi, 4 \pi]$, $\ldots,[k \pi-2 \pi, k \pi]$. Интеграл по каждому из этих отрезков неотрицателен, поэтому и выражение (16) неотрицательно. Если при этом $h_{\alpha}((k \pi-\alpha) / \sigma)=0$, то функция $\psi$ линейна на этих отрезках (и если $0<\alpha<\pi$, то равна нулю на $[0,2 \pi]$ ), а значит, $g$ представима в виде (15), где $g_{1}$ имеет вид (7).

Если $k \in \mathbb{Z}, k \leqslant 0$, то доказательство аналогично случаю $k \in \mathbb{N}$. Отметим только, что из равенства $h_{\alpha}((k \pi-\alpha) / \sigma)=0$ вытекает представление $(15)$, где $g_{1}$ имеет вид (10) или (12) соответственно в случаях, когда $k$ нечетное или четное. 
СЛЕДСТВИЕ 4. Пусть функиия $g \in L(0, \sigma)$, не убъвает, выпукла вниз и $g(t) \not \equiv 0$ на $(0, \sigma), g(+0)=0,0 \leqslant \alpha<\pi$. Тогда все нули функиии

$$
h_{\alpha}(x):=\int_{0}^{\sigma} g(t) \cos (t x+\alpha) d t
$$

вещественные и простые, при этом они расположены по одному в каждом из ниже перечисленных отрезков и только в них: если $0 \leqslant \alpha<\pi / 2$, то в отрезках

$$
\left[\frac{k \pi-\alpha}{\sigma}, \frac{k \pi+\pi / 2-\alpha}{\sigma}\right], \quad k \leqslant-1, \quad u \quad\left[\frac{k \pi-\pi / 2-\alpha}{\sigma}, \frac{k \pi-\alpha}{\sigma}\right], \quad k \geqslant 1,
$$

а если $\pi / 2 \leqslant \alpha<\pi$, то в отрезках

$$
\left[\frac{k \pi-\alpha}{\sigma}, \frac{k \pi+\pi / 2-\alpha}{\sigma}\right], \quad k \leqslant 0, \quad u \quad\left[\frac{k \pi-\pi / 2-\alpha}{\sigma}, \frac{k \pi-\alpha}{\sigma}\right], \quad k \geqslant 2,
$$

(во всех случаях $k \in \mathbb{Z})$. Число $(k \pi+\pi / 2-\alpha) / \sigma, k \in \mathbb{Z}$, является нулем только в случае $k=0, \alpha=\pi / 2$. Если число $(k \pi-\alpha) / \sigma, k \in \mathbb{Z}$, является нулем, то $g$ представима в виде (15), а $k \notin\{ \pm 1,0,2\}$, если $0<\alpha<\pi$ или $k \notin\{ \pm 1,0\}$, если $\alpha=0$.

ДокАЗАТЕЛЬСтво. Надо применить леммы 1, 2 и теоремы 1, 2. Следует только учесть, что $g \notin M_{\alpha}(\sigma)$ для всех $\alpha \in[0, \pi)$, и воспользоваться тождествами $h_{\alpha} \equiv f_{\alpha+\pi / 2}$ при $0 \leqslant \alpha<\pi / 2$ и $h_{\alpha} \equiv-f_{\alpha-\pi / 2}$ при $\pi / 2 \leqslant \alpha<\pi$. Если $h_{\alpha}((k \pi-\alpha) / \sigma)=0$, $k \in \mathbb{Z}$, то по лемме $2 g$ представима в виде $(15)$, где $g_{1} \in M_{\alpha}(\sigma)$. В этом случае $x h_{\alpha}(x)=g(\sigma-0) \sin (\sigma x+\alpha)-\int_{0}^{\sigma} g_{1}(t) \sin (t x+\alpha) d x$ и осталось применить теоремy 2.

СлЕДСТВИЕ 5. Пусть функция $g \in L(0, \sigma)$, не убывает, выпукла вниз и $g(t) \not \equiv 0$ на $(0, \sigma), a$

$$
h(x):=\int_{0}^{\sigma} g(t) \cos t x d t
$$

Тогда выполнены следующие утверждения.

1) Имеет место оиенка

$$
(-1)^{k} h\left(\frac{k \pi}{\sigma}\right) \geqslant 0 \quad \forall k \in \mathbb{Z} \backslash\{0\} .
$$

$\operatorname{Ecли~} h(k \pi / \sigma)=0$ при некотором $k \in \mathbb{Z} \backslash\{0\}$, mо $k \neq \pm 1$ u $g(t)-g(+0)$ представима в виде (15), где $g_{1} \in M_{0}(\sigma)$, а нуль $k \pi / \sigma$ имеет кратность 2 , если $g(\sigma-0)=0$ или является простым, если $g(\sigma-0) \neq 0$.

2) Если $h(0) \geqslant 0$, то функиия $h$ удовлетворяет условиям теоремы 1 , и все ее нули вещественные и простые, кроме случая $h(0)=0$, когда корень $x=0$ имеет кратность 2 .

3) Если $h(0)<0$, то функиия - $h$ удовлетворяет условиям следствия 2 и выполнены следующие утверэсдения:

а) если $g(\sigma-0) \leqslant 0$, то все нули функиии $h$ вещественные и простые, кроме, быть мохет, нулей вида $k \pi / \sigma, k \in \mathbb{Z} \backslash\{ \pm 1,0\}$ (см. утверэсдение 1$)$;

б) если $g(\sigma-0)>0$, то все нули функиии $h$ простые и среди них два чисто мнимые, а остальные вещественные. 
ДоКАЗАТЕЛЬСТво. 1) В силу леммы 2

$$
(-1)^{k} h\left(\frac{k \pi}{\sigma}\right)=(-1)^{k} \int_{0}^{\sigma}(g(t)-g(+0)) \cos \frac{k \pi t}{\sigma} d t \geqslant 0 \quad \forall k \in \mathbb{Z} \backslash\{0\},
$$

и если имеет место равенство, то $g(t)-g(+0)$ представима в виде $(15)$. В этом случае $x h(x)=g(\sigma-0) \sin \sigma x-\int_{0}^{\sigma} g_{1}(t) \sin t x d x$, где $g_{1} \in M_{0}(\sigma)$ и осталось применить теоремy 2 .

$2)$ Если $h(0) \geqslant 0$, то функция $h$ удовлетворяет условиям теоремы 1 , и значит, все ее нули вешественные. Так как $g(\sigma-0)>0$, то в силу теоремы 1 и утверждения 1 у функции $h$ кратных нулей нет, кроме случая $h(0)=0$, когда корень $x=0$ имеет кратность 2 .

$3)$ Если $h(0)<0$, то функция $-h$ удовлетворяет условиям следствия 2 , и значит, все ее нули вещественные, кроме, быть может, двух чисто мнимых простых нулей. Если $g(\sigma-0) \leqslant 0$, то $h(i x) \rightarrow-\infty$ при $x \rightarrow+\infty$ и поэтому мнимых нулей нет (в противном случае их число с учетом кратности не меньше 4$)$. Если $g(\sigma-0)>0$, то $h(i x) \rightarrow+\infty$ при $x \rightarrow+\infty$ и поэтому мнимые нули есть. Осталось применить следствие 2 и первое утверждение.

3. Целая функция $F$ называется устойчивой (см. [4]), если она не имеет нулей в замкнутой полуплоскости $\operatorname{Re} z \geqslant 0$. Пусть $F(z)=G(-i z)+i H(-i z)$, где $G$ и $H-$ целые функции, принимающие вещественные значения на вещественной прямой. Тогда функция $F$ называется сильно устойчивой, если существует $\varepsilon>0$ такое, что функция $G(-i z)+(i+\zeta) H(-i z)$ устойчива при каждом $\zeta \in \mathbb{C}$ таком, что $|\zeta|<\varepsilon$ (см. [4, c. 129], а также $[5$, гл. IV]). Очевидно, что любая сильно устойчивая функция является устойчивой.

ТЕОрема ЧеБотарёва [4], [5]. Функция $F$ сильно устойчива тогда и только тогда, когда выполнены следуюшие условия:

1) $G$ и не имеют общих нулей и для всех $\lambda, \mu \in \mathbb{R}, \lambda^{2}+\mu^{2} \neq 0$, функиия $\lambda G+\mu H$ не имеет нулей в $\mathbb{C} \backslash \mathbb{R} ;$

2) неравенство

$$
G(x) H^{\prime}(x)-G^{\prime}(x) H(x)>0
$$

выполняется хотя бы для одного значения $x_{0} \in \mathbb{R}$.

Кроме того, при выполнении условий 1) и 2) указанное неравенство выполняется для всех $x \in \mathbb{R}$.

ТЕОремА 3. Пусть функиия $g \in L(0, \sigma), g(x) \geqslant 0, g(x) \not \equiv 0$, не убывает на $(0, \sigma) u$

$$
F(z):=\int_{0}^{\sigma} g(t) e^{z t} d t, \quad G(z):=\int_{0}^{\sigma} g(t) \cos z t d t, \quad H(z):=\int_{0}^{\sigma} g(t) \sin z t d t .
$$

Тогда

1) если $g \notin \bigcup_{0 \leqslant \alpha<\pi} M_{\alpha}(\sigma)$, то $F$ сильно устойчива и имеет место неравенство

$$
G(x) H^{\prime}(x)-G^{\prime}(x) H(x)>0, \quad x \in \mathbb{R}
$$

2) если $g \in \bigcup_{0 \leqslant \alpha<\pi} M_{\alpha}(\sigma)$, mo

а) все нули функиии $F$ находятся в полуплоскости $\operatorname{Re} z \leqslant 0$; 
б) на прямой $\operatorname{Re} z=0$ лежит бесконечно много ее нулей и все они простые;

в) число прямых $\operatorname{Re} z=c, c \leqslant 0$, на которых лежст нули $F$, конечно и на каждой из них лежит бесконечно много ее нулей;

г) имеет место неравенство

$$
G(x) H^{\prime}(x)-G^{\prime}(x) H(x) \geqslant 0, \quad x \in \mathbb{R},
$$

которое обрашается в равенство в бесконечном числе точек из $\mathbb{R}$.

ДоказАТЕЛЬСтво. 1) Очевидно, $G$ и $H$ - целые вещественные функции и $F(z)=$ $G(-i z)+i H(-i z)$. Рассмотрим

$$
f_{\alpha}(z):=\int_{0}^{\sigma} g(t) \sin (t z+\alpha) d t=\sin \alpha G(z)+\cos \alpha H(z) .
$$

По теореме 2 все нули функции $f_{\alpha}$ вешественные и простые при всех $\alpha \in[0, \pi)$ (и значит, при всех $\alpha \in \mathbb{R}$ ). Отсюда сразу следует, что $G$ и $H$ не имеют общих нулей. Действительно, если $G\left(x_{0}\right)=H\left(x_{0}\right)=0$ при некотором $x_{0}$, то $x_{0} \in \mathbb{R}$ и $G^{\prime}\left(x_{0}\right) \neq 0$. Тогда при $\alpha=\operatorname{arctg}\left(-H^{\prime}\left(x_{0}\right) / G^{\prime}\left(x_{0}\right)\right)$ число $x_{0}$ является для $f_{\alpha}$ нулем кратности не менее, чем 2 , что противоречит доказанному. Осталось применить теорему Чеботарёва, при этом следует учесть, что

$$
G(0) H^{\prime}(0)-G^{\prime}(0) H(0)=\int_{0}^{\sigma} g(t) d t \int_{0}^{\sigma} g(t) t d t>0
$$

2) Функция $g_{n}$, определяемая равенством (14), удовлетворяет условиям теоремы 3 и, очевидно, $g_{n} \notin M_{\alpha}(\sigma)$ для всех $\alpha \in[0, \pi)$. По доказанному все нули соответствующей функции $F_{n}$ лежат в полуплоскости $\operatorname{Re} z<0$, и значит, нули предельной функции $F \not \equiv 0$ лежат в замкнутой полуплоскости $\operatorname{Re} z \leqslant 0$.

Из определения класса $M_{\alpha}(\sigma)$ вытекает, что при некотором $k \in \mathbb{Z} \backslash\{0\}$ функция $g$ представима одним из равенств $(5),(7),(10),(12)$, а соответствуюшие точки $x_{p}, 0 \leqslant p \leqslant$ $m(k)$, образуют арифметическую прогрессию с разностью $d:=(2 \pi \sigma /(k \pi-\alpha)) \operatorname{sign} k>$ 0 . Учитывая, что $x_{0}-x_{-1}=d$ при $k=2 j, j \in \mathbb{N}, \alpha=0$, а в остальных случаях $c_{0}=0$, получаем

$F(z)=\sum_{p=0}^{m(k)} \int_{x_{p-1}}^{x_{p}} g(t) e^{z t} d t=\sum_{p=0}^{m(k)} c_{p} z^{-1}\left(e^{z x_{p}}-e^{z x_{p}-1}\right)=\frac{e^{z d}-1}{z} e^{z x_{0}} \sum_{p=0}^{m(k)} c_{p} e^{z d(p-1)}$.

Из (19) видно, что числа вида $i 2 \pi n d^{-1}, n \in \mathbb{Z} \backslash\{0\}$, являются нулями функции $F$. Все нули $F^{\prime}$ лежат в полуплоскости $\operatorname{Re} z<0$ (надо утверждение 1 применить к $g_{1}(t)=$ $g(t) t)$. Поэтому все нули $F$, лежащие на мнимой оси, простые. Утверждение в) сразу следует из (19), если учесть, что не равньй тождественно нулю алгебраический многочлен имеет конечное число нулей. Неравенство (18) получается предельным переходом из (17). То, что оно обрашается в равенство в бесконечном числе точек из $R$, вытекает из соотношения $|F(i x)|^{2}=G^{2}(x)+H^{2}(x), x \in \mathbb{R}$. 
ЗАмЕчаниЕ. Условие $g \in \bigcup_{0 \leqslant \alpha<\pi} M_{\alpha}(\sigma)$ эквивалентно двум условиям:

1) $g(x) \geqslant 0$ и не убывает на $(0, \sigma)$;

2 ) при некотором $x_{0} \in[0, \sigma)$ функция $g$ является на $\left(x_{0}, \sigma\right)$ кусочно постоянной с равностоящими узлами (т.е. интервал $\left(x_{0}, \sigma\right)$ можно разбить на конечное число интервалов одинаковой длины, на каждом из которых $g$ постоянна) и $g(x) \equiv 0$ на $\left(0, x_{0}\right)$, если $x_{0}>0$.

Действительно, если функция представима одной из формул $(5),(7),(10),(12)$, то она, очевидно, удовлетворяет условиям 1) и 2). И наоборот, если $g$ удовлетворяет условиям 1$), 2)$ и $g(x) \not \equiv 0$ на $(0, \sigma)$, то у функции $F$ из теоремы 3 имеются нули на прямой $\operatorname{Re} z=0\left(F\right.$ имеет вид (19)) и, значит, $g \in \bigcup_{0 \leqslant \alpha<\pi} M_{\alpha}(\sigma)$.

Функция $h: \mathbb{R} \rightarrow \mathbb{C}$ называется положительно определенной на $\mathbb{R}$, если для всех $n \in \mathbb{N}$, всех $\left\{x_{k}\right\}_{k=1}^{n} \subset \mathbb{R}$ и всех $\left\{c_{k}\right\}_{k=1}^{n} \subset \mathbb{C}$ выполняется неравенство

$$
\sum_{k, j=1}^{n} c_{k} \bar{c}_{j} h\left(x_{k}-x_{j}\right) \geqslant 0 \text {. }
$$

Если $h \in C(\mathbb{R}) \cap L(\mathbb{R})$, то по теореме Бохнера-Хинчина положительная определенность $h$ эквивалентна неотрицательности преобразования Фурье, т.е.

$$
\hat{h}(x):=\int_{-\infty}^{+\infty} h(t) e^{-i t x} d x \geqslant 0, \quad x \in \mathbb{R} .
$$

СлЕДСТВИЕ 6. Пусть $g \in L(0, \sigma), g(x) \geqslant 0$ не убивает на $(0, \sigma)$, а четная функиия $h$ определяется равенствами

$$
h(x):=0 \quad \text { npu } \quad x \geqslant \sigma \quad u \quad h(x):=\int_{x}^{\sigma}(2 u-x) g(u) g(u-x) d u, \quad 0 \leqslant x<\sigma .
$$

Тогда $h \in L(\mathbb{R}), \hat{h}(x) \geqslant 0, x \in \mathbb{R}$, и уравнение $\hat{h}(x)=0, x \in \mathbb{R}$, имеет решение тогда и только тогда, когда $g \in \bigcup_{0 \leqslant \alpha<\pi} M_{\alpha}(\sigma)$. Если дополнительно $g \in L_{2}(0, \sigma)$, то функиия $h$ является полохительно определенной на $\mathbb{R}$.

ДокАЗАТЕльСТво. Если $g$ продолжить нулем на $(\sigma,+\infty)$, то, используя связь между преобразованием Фурье и сверткой, получаем

где

$$
G(x) H^{\prime}(x)-G^{\prime}(x) H(x)=\frac{1}{4} \hat{h}_{0}(x),
$$

$$
h_{0}(x)=\int_{-\infty}^{+\infty} g(|u|) g(|x-u|)(x-u)(\operatorname{sign}(x-u)-\operatorname{sign} u) d u
$$

Нетрудно показать, что $h_{0}(x)=2 h(x), x \in \mathbb{R}$. Осталось учесть, что свертка двух функций из $L(\mathbb{R})$ есть функция из $L(\mathbb{R})$, и применить неравенства $(17),(18)$. Если дополнительно $g \in L_{2}(0, \sigma)$, то $h \in \mathbb{C}(\mathbb{R})$, и можно применить теорему Бохнера-Хинчина.

\section{СПИСОК ЦИТИРОВАННОЙ ЛИТЕРАТУРЫ}

[1] Polya G. Über die Nullstellen gewisser ganzer Funktionen // Math. Z. 1918. V. 2. P. 352-383.

[2] Ахиезер Н. И. Лекции по теории аппроксимации. М.: Наука, 1965.

[3] Чеботарёв Н. Г., Мейман Н. Н. Проблема Рауса-Гурвица для полиномов и целых функций // Тр. МИАН. 1949. Т. 26.

[4] Постников М. М. Устойчивые многочлены. М.: Наука, 1981.

[5] Седлецкий А. М. О целых функциях класса С. Н. Бернштейна, не являющихся преобразованиями Фурье-Стилтьеса // Матем. заметки. 1997. Т. 61. № 3. С. 367-380. 